Journal of Animal and Veterinary Advances 10 (11): 1462-1468, 2011

ISSN: $1680-5593$

(C) Medwell Journals, 2011

\title{
The Association of ANKRD2 with Loin Depth and Muscle Firmness in Pigs
}

\author{
Ling Sun, Xinjie Dong, Bin Fan and Bang Liu \\ Lab of Molecular Biology and Animal Breeding, \\ Key Laboratory of Agricultural Animal Genetics, Breeding and Reproduction of Ministry of Education, \\ Key Laboratory of Swine Genetics and Breeding of Ministry of Agriculture, \\ Huazhong Agricultural University, 430070 Wuhan, P.R. China
}

\begin{abstract}
The Muscle Ankyrin Repeat Proteins (MARPs) family is essential for cardiogenesis and Cardiac hypertrophy (CARP), skeletal muscular stretch and development (ANKRD2/ARPP) and during recovery following starvation (DARP). Here we describe the isolation and molecular characterization of the full-length cDNA of porcine gene $A N K R D 2$ (ankyrin repeated domain 2). The cDNA sequence and predicted protein sequence shared high sequence identity with other mammalian counterparts. The Reverse TranscriptasePolymerase Chain Reaction (RT-PCR) revealed that ANKRD2 has a high level of expression in skeletal muscle and a low expression in heart while the other tissues being tested had no expression. The expression of $A N K R D 2$ gene was up-regulated accompanying with the different embryo developmental stages (embryo 33 , 65, 90 days; postnatal 2 days, adult) and reached the peak at the adult time. A T/C single nucleotide polymorphism in ANKRD2 exon5 was detected and a HaeIII PCR-Restriction Fragment Length Polymorphism (PCR-RFLP) was then developed. Association of the genotypes with growth and carcass traits revealed significant associations of the porcine ANKRD2 loin depth $(\mathrm{p}<0.05)$ and firmness $(\mathrm{p}<0.05)$.
\end{abstract}

Key words: Sequence analysis, expression profile, association analysis, carcass trait, meat quality trait, ANKRD2

\section{INTRODUCTION}

The Ankyrin-Repeat Domain 2 (ANKRD2) was first identified in response to mechanical stretch of mouse skeletal muscles and has been reported as a member of a family of Muscle Ankyrin Repeat Proteins (MARPs) (Kemp et al., 2000). Human ANKRD2 is mainly expressed in normal adult skeletal muscle and low expressed in heart and it is also expressed in different slow fiber with solely characterized expression level (Pallavicini et al., 2001). In human fetus, the expression levels in skeletal muscle and cardiac muscle are very low or only a trace level (Moriyama et al., 2001). In response to stress signals, ANKRD2 is expressed at a higher level after muscle stretching and could combine with titin, myopalladin and calpain protease p94 to regulate expression of the related genes (Kojic et al., 2004). In TWEAK-induced skeletal muscle wasting mice, ANKRD2 is down-regulated (Panguluri et al., 2010) but in contrast its expression is severely increased in human cardiac hypertrophy (Pallavicini et al., 2001). ANKRD2 protein is localized in the sarcomere I band and conditionally translocated into the nucleus in response to muscular damage (Kojic et al., 2004; Tsukamoto et al., 2008). It has been proved that ANKRD2 can interact with three transcriptional factors: YB-1, p53 and PML and consequently was co-localized in the nucleus bodies and enhanced the p53 up-regulation of the p21 promoter. Researches about the p53 and p21 functions revealed the crucial role for adipocyte differentiation and hypertrophy (Hallenborg et al., 2009; Inoue et al., 2008).

During vertebrate myogenesis various transcription factors are coordinated expressed to control the specification of the muscle which is origin from the mesodermal precursor cells and the differentiation of committed myoblast into the fusion fibers. The transcription factor MyoD belong to basic Helix-loopHelix family plays a key role in the progress and primarily is targeted to muscle gene regulation (Molkentin and Olson, 1996). A recent study suggests that ANKRD2 is a downstream gene whose promoter contains a functional region for $\mathrm{MyoD}$ and during the myoblast differentiation

Corresponding Author: Bang Liu, Lab of Molecular Biology and Animal Breeding,

Key Laboratory of Agricultural Animal Genetics, Breeding and Reproduction of Ministry of Education, Key Laboratory of Swine Genetics and Breeding of Ministry of Agriculture,

Huazhong Agricultural University, 430070 Wuhan, P.R. China 
it is up-regulated (Bean et al., 2005). The previous results suggested the contribution of ANKRD2 to the proliferation and apoptosis balance during myogenic differentiation in vitro (Bean et al., 2008; Tsukamoto et al., 2008). Considering of the characters about the regulated region and expressed condition of ANKRD2, it is convinced that ANKRD2 is essential for myogenic differentiation.

In the present study, the porcine $A N K R D 2$ gene full-length cDNA combined with its expression characterization were studied and analyzed in order to depict a clear function pattern about the gene in muscle. Scanning, genotyping and association analysis of SNP in the gene were carried out in a RFI selection experiment in a Yorkshire pig population with the purpose to identify new genetic markers for markers assistant selection. This research will contribute to a better understanding of the molecular mechanisms of ANKRD2 gene during muscle development.

\section{MATERIALS AND METHODS}

Isolation of full-length cDNA of porcine $A N K R D 2$ gene: The sequences of cDNAs of human ANKRD2 gene (GenBank: NM_020349) was used to search the pig EST databases by BLAST (http:/www.ncbi.nlm.nih.gov/ blast/). Porcine EST (11 for ANKRD2) which shared at least $90 \%$ identity to the corresponding human cDNAs were assembled into contigs for primer design. Genespecific primers were shown in Table 1. The porcine ANKRD2 full-length cDNA was obtained by the Rapid Amplification of cDNA Ends (RACE). The RNA was extracted from the muscle tissue of a Landrace (Hubei Province) with a TRIzol reagent kit (Life Technologies, Grand Island, NE, USA). RACE was performed according to the instructions of the SMARTTM RACE cDNA Amplification Kit (Clontech Inc, Palo Alto, CA, USA). The PCR products were purified with Gel Extraction Mini Kit (Waston Biotechnologies Inc, Shanghai, China) and cloned into the pMD18-T vector (Takara, Dalian, China), then sequenced using a commercial service. ORF and the amino acid sequences of porcine $A N K R D 2$ gene were deduced with the program Seqman (DNA star, Madison, WI, USA). The DNA sequences were compared using the BLAST program from the National Center for Biotechnology Information (NCBI). The nucleotides and amino acid sequences used have been deposited at GenBank $^{\mathrm{TM}} / \mathrm{EBI}$ Data Bank.

Expression profile analysis: The Reverse TranscriptasePolymerase Chain Reaction (RT-PCR) method was applied to detect the expression of the gene. Total RNAs were extracted from adult porcine of a landrace heart, liver, spleen, lung, kidney, skeletal muscle, intestine, brain, lymph node and testis. In addition RNAs were extracted from skeletal muscles at different stages: 33, 65, 90 days embryos, postnatal 4 days and adult Tong cheng pigs. Reverse transcriptions were performed as described by Qiu et al. (2008). The primer pairs P2L and P2R (Table 1) were used to detect the expression levels. PCR conditions were $4 \mathrm{~min}$ at $95^{\circ} \mathrm{C}$ followed by 28 cycles of $30 \mathrm{sec}$ at $94^{\circ} \mathrm{C}, 30 \mathrm{sec}$ at $61^{\circ} \mathrm{C}, 20 \mathrm{sec}$ at $72^{\circ} \mathrm{C}$ and a final extension of $5 \mathrm{~min}$ at $72^{\circ} \mathrm{C}$. Amplification of GAPDH was performed as an internal control in the same conditions. Finally $8 \mu \mathrm{L}$ of each PCR products were used for the expression profile analysis on $2.0 \%$ agarose gels.

Real-time PCR: The expression level of $A N K R D 2$ gene in three developmental stages was detected by SYBR Green I assay. Each real-time PCR (in $20 \mu \mathrm{L}$ ) reaction contained $1 \times$ PCR buffer (TaKaRa), $2.0 \mathrm{mM} \mathrm{MgCl}_{2}, 500 \mu \mathrm{M}$ each dNTP, $0.4 \mu \mathrm{M}$ primers (Table 1 ), $0.3 \times$ SYBR Green I and 1 $\mathrm{U}$ Taq DNA polymerase (TaKaRa) plus $2 \mu \mathrm{L}$, normalized template cDNA. The cycling conditions consisted of an initial, single cycle for $3 \mathrm{~min}$ at $95^{\circ} \mathrm{C}$ followed by 35 cycles of cycling consisting of $15 \mathrm{sec}$ at $94^{\circ} \mathrm{C}, 20 \mathrm{sec}$ at $61^{\circ} \mathrm{C}$, $20 \mathrm{sec}$ at $72^{\circ} \mathrm{C}$ and fluorescence acquisition at $82^{\circ} \mathrm{C}$ for $1 \mathrm{sec}$. The specific PCR products were confirmed by

Table 1: Primer pairs for porcine ANKRD2

\begin{tabular}{|c|c|c|c|}
\hline Primer name & Primer sequence $\left(5^{\prime}-3^{\prime}\right)$ & Size (bp) & $\mathrm{TM}\left({ }^{\circ} \mathrm{C}\right.$ \\
\hline ANKRD2 3'RACE PF & 5' TGGACATCAATGCCAAAGACAGAGA 3' & 423 & 66 \\
\hline ANKRD2 5'RACE PR & 5' CCATCCGCCAGGAACTTCTCAAT 3' & 423 & 70 \\
\hline ANKRD2 5'NEST PR & 5' CCGCCGCTTTCAGGAATGTCT $3 '$ & 384 & 66 \\
\hline ANKRD2 semi-RT-PCR-PF & 5' AGGATGAGAAGCACCACAGGC3' & 321 & 61 \\
\hline ANKRD2 semi-RT-PCR PR & 5' GGAACTGGTCGCAGGTGTCT3' & & \\
\hline GAPDH semi-PR-PCR-PF & 5' CCTTCATTGACCTCCACTAC3' & 320 & 61 \\
\hline GAPDH semi-PR-PCR-PR & 5' GTTGTCATACTTCTCATGGTTC3' & & \\
\hline ANKRD2 QPCR-PF & 5' TCATCAAACTGCTGCTCCTACA 3' & 144 & 61 \\
\hline ANKRD2 QPCR-PR & 5' GCCCGTTCTGCTCCGACT 3' & & \\
\hline 18S Ribosomal RNA-PF & 5' TTTCGCTCTGGTCCGTCTTG 3' & 102 & 61 \\
\hline 18S Ribosomal RNA-PR & 5' ATCATGGCCTCAGTTCCGAA 3' & & \\
\hline ANKRD2 SNP1-PF & 5' GGTTAAGCCCTGACAGTCTCTCT 3' & 238 & 57 \\
\hline ANKRD2 SNP1-PR & 5' CCCCAAGCCACCTGTTTCT 3' & & \\
\hline
\end{tabular}


melting curve analysis. cDNAs from three fetus muscle samples in each stage were used to detect the expression changes of the target gene and all PCRs were performed in triplicate and gene expression levels were quantified relatively to the expression of $18 \mathrm{~S}$ ribosomal RNA using Gene Expression Macro software (Bio-Rad, Richmond, CA, USA) by employing an optimized comparative $\mathrm{C}^{\mathrm{t}}$ (ddCt) value method. Expression levels were considered not detectable when the $\mathrm{C}^{\mathrm{t}}$ value of the targeted gene exceeded 35 in the sample tissue. The t-test was conducted to identify genes differing in expression, $\mathrm{p}<0.05$ was considered significant.

SNP identification: One pair of primers (SNP-PF and SNP-PF in Table 1) on exon 5 were used to scan the Single Nucleotide Polymorphism (SNP) using the genomic DNA pool from Tongcheng, Erhualian and Duroc pigs. The PCR reactions were performed in a volume of $20 \mu \mathrm{L}$ containing $50 \mathrm{ng}$ of DNA, $1^{\circ} \mathrm{C}$ PCR buffer, $0.3 \mu \mathrm{m}$ of each primer, $75 \mu \mathrm{m}$ of each $\mathrm{dNTP}, 1.5 \mathrm{mM}$ of $\mathrm{MgCl}_{2}$ and $1 \mathrm{U}$ of Taq DNA polymerase (Yeli, Bioscience Co. Ltd, Shanghai, China). The $\mathrm{PCR}$ reaction conditions were $5 \mathrm{~min}$ at $95^{\circ} \mathrm{C}$ followed by 34 cycles of $40 \mathrm{sec}$ at $94^{\circ} \mathrm{C}, 30 \mathrm{sec}$ at $57^{\circ} \mathrm{C}, 40 \mathrm{sec}$ at $72^{\circ} \mathrm{C}$ and a final extension of $5 \mathrm{~min}$ at $72^{\circ} \mathrm{C}$. The PCR products were pooled and sequenced. The polymorphism site was analyzed by sequence comparisons using the DNAstar software (DNAstar Inc.) and was further identified by the PCR-Restriction Fragment Length Polymorphism (PCR-RFLP) method.

Association analysis of the porcine $A N K R D 2$ gene with economic traits: The association analysis of $A N K R D 2$ gene was performance in Yorkshire pigs. The experiment population were composed of 171 purebred Yorkshire gilts from a line selected for lower RFI (Residual Feed Intake, $\mathrm{n}=79)$ and a randomly selected control line $(\mathrm{n}=92)$ which were sampled from the second parity of the fifth generation of a selection experiment for RFI at Iowa State University. Experimental protocols for this study were approved by the Iowa State University Institutional Animal Care and Use Committee (Cai et al., 2008). Analyzed traits consisted of RFI, growth rate, carcass composition and meat quality traits described as follows:

Intramuscular Fat (IMF), 10th rib back fat depth (OFFBF), loin muscle area (OFFLMA), Loin depth (LEMM), Hot Carcass Weight (HCW), Back Fat Depth (BFD), Loin Muscle Depth (LMD), drip loss, Water Centrifugation Loss (WCL), $\mathrm{pH}$ at 2 days postmortem $(\mathrm{pH})$, Hunter value (L) for meat color, Water holding capacity, Firmness, wetness, Color and marbling scores, Intramuscular Lipid (IML), moisture (IMM) content, moisture lost after cooking (cook loss), juiciness, tenderness and off flavor. The basic statistics including genotype frequency, allele frequency and a $\chi^{2}$-test for differences of genotype distributions between selection and control lines were computed using the FREQ procedure of SAS 9.0 (SAS Institute, Cary, NC, USA). The association analyses between genotypes and traits were performed using the MIXED procedure of SAS 9.0 (SAS Institute, release 9.1, Cary, NC, USA), with fixed and random effects and covariates differing by trait. For the traits RFI and ADG, the model was as follows:

$$
\begin{gathered}
\mathrm{Y}=\mu+\text { Group }+ \text { Line }+ \text { SNP genotype }+ \text { SNP } \\
\text { genotype }{ }^{*} \text { line }+ \text { Pen }+ \text { Dam }+\mathrm{e}
\end{gathered}
$$

Group, line, SNP genotype and SNPgenotype* line were fixed effects; pen and dam were random effects. There was no covariate in the model because RFI and ADG were estimated on the standard test period from 90-210 days old. For other traits, the model was the same as that for RFI analysis except that BW at Off-test (OFFBW) was used as a covariate in the model. In this study significant effects were those with $\mathrm{p}<0.05$ and suggestively significant effects were those with $\mathrm{p}<0.1$.

\section{RESULTS AND DISCUSSION}

Molecular cloning and sequence analysis of porcine $A N K R D 2$ gene: The SMART RACE protocol allowed the isolation of full-length cDNA of $1174 \mathrm{bp}$ (Gene Bank accession HQ403605-HQ403607). It contained an Open Reading Frame (ORF) of 987 bp flanked by a 5' untranslated region of $27 \mathrm{bp}$. The $3^{\prime}$ flanking region contained a canonical polyadenylation signal AATAAA ending 13 nucleotides upstream from the poly (A) segment. The porcine ANKRD2 cDNA sequence encoded 328 amino acids with a calculated molecular mass of $36.7 \mathrm{kDa}$ and isoelectric point of 6.25 . The amino acids displayed a 90 and $89 \%$ identity with human and mouse. The putative domains, motifs and features of porcine ANKRD2 protein were predicted by Scan-Prosite. The proteins were possibly contained four ankyrin repeat profile (PS50088, residues 149-181, 182-214, 215-247, 248-280). One cAMP and cGMP dependent protein kinase phosphorylation site (PSO0004, residues 65-68), three casein kinaseII phosphorylation site (PSO0006), seven $\mathrm{N}$-myristoylation site (PSO0008), two protein kinase C phosphorylation site (PSO0005). Cytoplasmic/nuclear discrimination predicted porcine ANKRD2 protein by PSORTII and WoLF PSORT. The protein might both in the nuclear and cytoplasm. Prediction of protein sorting signals identified two pat 4 nuclear localization motifs RKKR and KKRK (Hicks and Raikhel, 1995). There were 


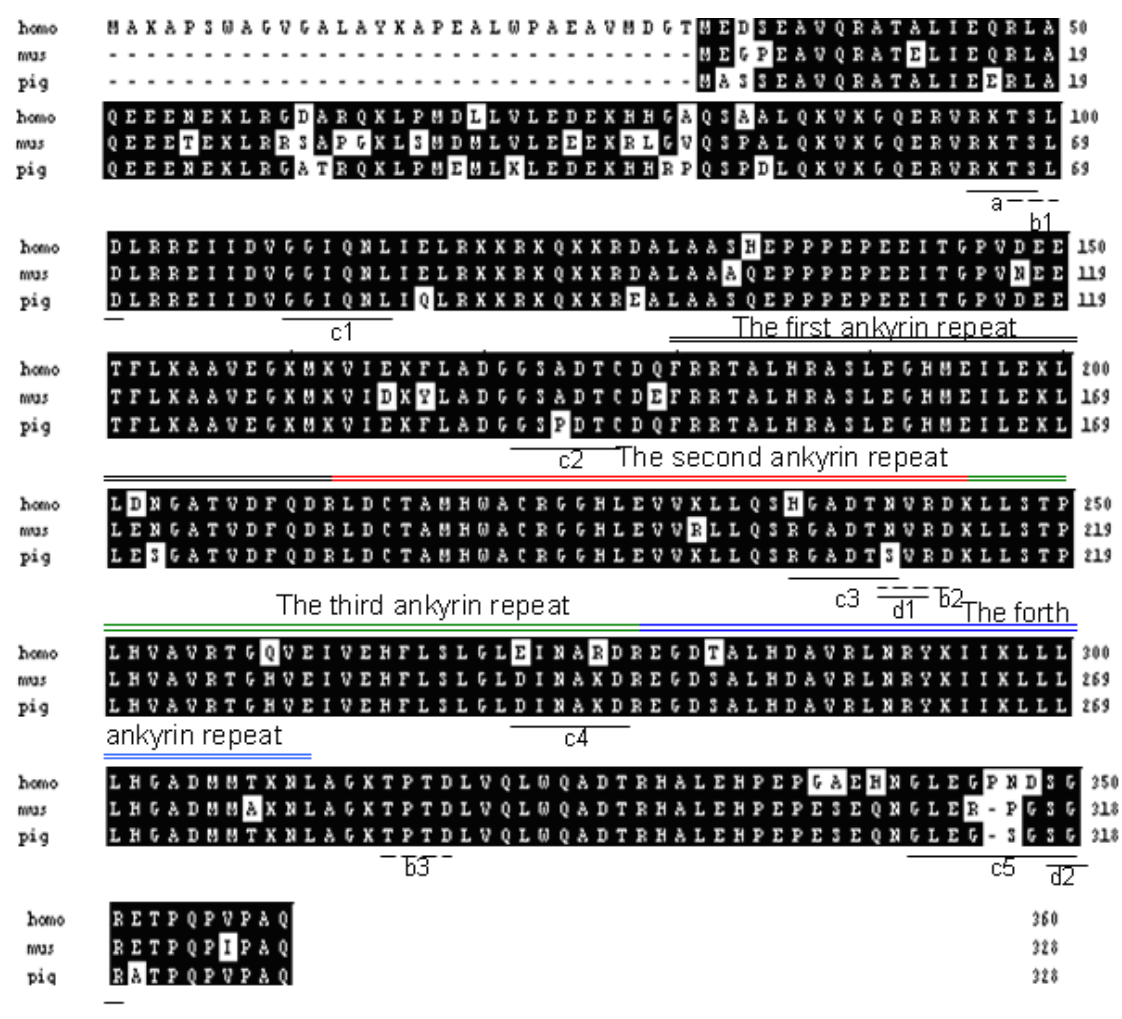

Fig. 1: Multiple alignment of different mammalian ANKRD2 peptide sequences. The deduced amino acid sequences of the porcine ANKRD2 are shown aligned to those from human (NP_065082) and mouse (NP_064417). Identical amino acid residues among species are presented with a black background. Four ankyrin repeat profile (PS50088) was predicted at positions $149-280$ of the porcine sequence. This motif is double-marked above the sequences. Underlined amino acids indicate patterns with a high probability of occurrence: $a=c A M P$ and cGMP-dependent protein kinase phosphorylation site (PS00004), b = Casein kinase II phosphorylation site (PSO0006), c = Nmyristoylation site (PSO0008), d = Protein kinase C phosphorylation site (PSO0005)

also predicted that porcine protein probability in cytoplasm however, it was a little lower than in nuclear. Another motif is the PEST sequence which serves as proteolysis signal that targets proteins for rapid destruction (Fig. 1).

\section{Porcine ANKRD2 RT-PCR and Realtine-PCR results:} RT-PCR analysis demonstrated that porcine ANKRD2 gene expressed predominant in skeletal muscles very low in heart, live and kidney (Fig. 2a) and expression result in skeletal muscle of different development stages showed no expression in 33 days embryo and up-regulated from 33 days embryo to adult in the five phases (include 65 days embryo, 90 days embryo, 4 days postnatal and adult) (Fig. 2b). Real-time result showed that pig ANKRD2 was significantly differentially expressed among these stages in both Tongcheng pigs $(p<0.01)$ and Landrace $(p<0.01)$. At $90 \mathrm{dpc}$, the real-time RT-PCR results showed that the expression level of $A N K R D 2$ gene of Tongcheng pig was significantly lower than that in Landrace ( $<0.01$ ) (Fig. 3).
SNP identification and Allele frequencies of the ANKRD2 gene in different pig breeds: One C534T SNP in the exon 5 was found and detected by digestion with HaeIII, resulting in a 238 bp PCR amplicon-produced allele $\mathrm{T}$ (76 and $164 \mathrm{bp}$ ) and allele $\mathrm{C}(76,124$ and $39 \mathrm{bp}$ ) (results were not showed). After HaeIII digestion, the allele distribution revealed that the Chinese indigenous breeds had higher frequencies of the $\mathrm{C}$ allele whereas the exotic breed Duroc pigs had higher frequency of the $\mathrm{T}$ allele (Table 2). The $\chi^{2}$ result for the allele frequency distribution indicated that there was a significant difference between Chinese indigenous pig and exotic breeds Duroc $(\mathrm{p}<0.01)$ (Table 3).

Association analysis of the porcine $A N K R D 2$ gene with economic traits: The trait association analysis in the Yorkshire population suggested that ANKRD2 C/T SNP was significantly association with LEMM $(\mathrm{p}<0.05)$ and Firmness $(\mathrm{p}<0.05)$ and pigs with TT genotype was obviously higher than pigs with $\mathrm{CC}$ and TC genotypes (Table 4). 


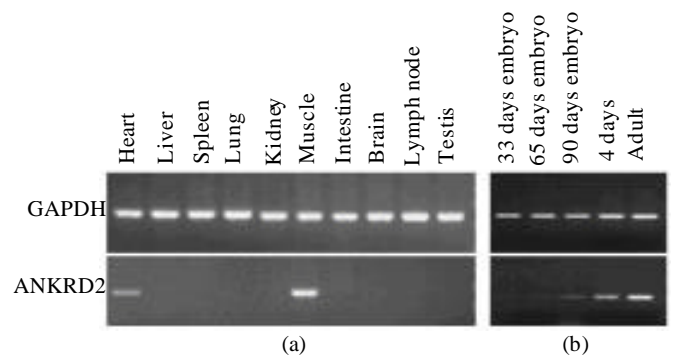

Fig. 2: The expression patterns of porcine ANKRD2 in 10 tissues and 5 different developmental stages of skeletal muscle. (a) Semi-quantification expression analyses for ANKRD2 and GAPDH (internal control) at different tissue as follows: heart, live, spleen, lung, kidney, muscle, intestine, brain, lymph node and testis of adult Landrace pig, respectively. (b) RT-PCR expression analyses for ANKRD2 and GAPDH (internal control) at different stages, including 33 days embryo, 65 days embryo, 90 days embryo, 4 days and adult Tongcheng pig, respectively

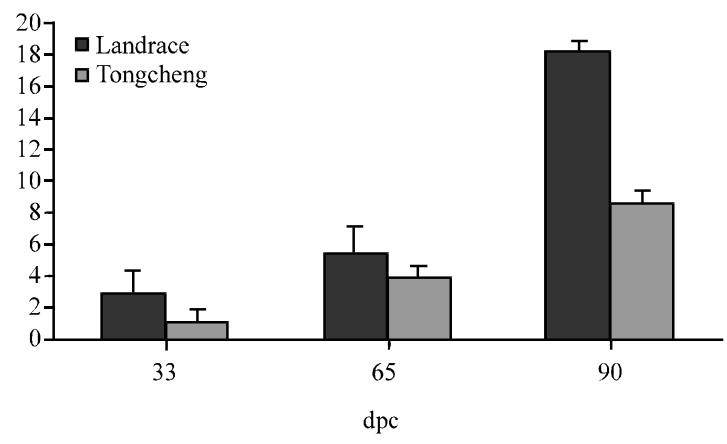

Fig. 3: The real-time PCR comparison of porcine ANKRD2 expression patterns in two pig breeds. The values shown in this figure are the averages of three calculated values from three independent experiments. Error bars represent the SD $(n=3)$ of relative mRNA expression levels of ANKRD2 to endogenous $18 \mathrm{~S}$ Ribosomal RNA. Blue columniation: 33 days embryo, 65 days embryo, 90 days of adult Landrace pig respectively. Red columniation: 33 days embryo, 65 days embryo, 90 days of adult Tongcheng pig respect. The dramatic increasing expression level in $90 \mathrm{dpc}$ Landrace was predominant higher than that in Tongcheng $(\mathrm{p}<0.05)$

In this study we cloned full length cDNA of pig $A N K R D 2$ gene, studied the expression pattern of ANKRD2 in pigs and evaluated the association of ANKRD2 polymorphism with IMF, LEMM and Firmness.
Table 2: Allele frequencies of the porcine $A N K R D 2$ gene in different breeds

\begin{tabular}{|c|c|c|c|c|c|c|}
\hline \multirow[b]{2}{*}{ Breed } & \multirow[b]{2}{*}{ Sample size } & \multicolumn{3}{|c|}{ Genotype } & \multicolumn{2}{|c|}{$\begin{array}{l}\text { Allele } \\
\text { frequency (\%) }\end{array}$} \\
\hline & & TT & $\mathrm{TC}$ & $\mathrm{CC}$ & $\mathrm{T}$ & $\mathrm{C}$ \\
\hline Duroc & 30 & 29 & 1 & 0 & 98.3 & 1.7 \\
\hline Meishan pigs & 19 & 0 & 9 & 10 & 23.6 & 76.4 \\
\hline Erhualian pigs & 29 & 5 & 11 & 13 & 36.2 & 63.8 \\
\hline Large black-white & 17 & 2 & 6 & 9 & 29.4 & 70.6 \\
\hline Qingping pigs & 31 & 5 & 10 & 16 & 32.3 & 67.7 \\
\hline Yushan black pigs & 28 & 8 & 10 & 10 & 46.4 & 53.6 \\
\hline
\end{tabular}

Table 3: $\chi^{2}$ test results for the allele frequency distribution among different populations of HaeII-RFLP for the porcine ANKRD2 gene

\begin{tabular}{|c|c|c|c|c|c|}
\hline Breeds & Duroc & $\begin{array}{l}\text { Meishan } \\
\text { pigs }\end{array}$ & $\begin{array}{l}\text { Erhualian } \\
\text { pig }\end{array}$ & $\begin{array}{c}\text { Large } \\
\text { black-white }\end{array}$ & $\begin{array}{c}\text { Qingping } \\
\text { pigs }\end{array}$ \\
\hline Meishan pigs & $45.2089^{* *}$ & - & - & - & - \\
\hline Erhualian pig & $25.5801^{* *}$ & $8.71724^{*}$ & - & - & - \\
\hline Large black-white & $35.1835^{* *}$ & 2.54939 & 4.00485 & - & - \\
\hline Qingping pigs & $40.2992^{* *}$ & 3.77467 & 4.31908 & 0.177479 & - \\
\hline Yushan black pigs & $29.2484^{* *}$ & $6.57014^{*}$ & 0.474666 & 2.08854 & 1.92937 \\
\hline
\end{tabular}

${ }^{*} \mathrm{p}<0.05 ;{ }^{* * *} \mathrm{p}<0.01 . \chi_{0.05}^{2}(\mathrm{df}=2)=5.99, \chi_{0.01}^{2}(\mathrm{df}=2)=9.21$

Table 4: The results for the association analysis of HaeIII-RFLP for the exon

\begin{tabular}{lcll}
\multicolumn{4}{c}{5 of porcine $A N K R D 2$} \\
\hline Genotype & No. of pig & LEMM & Firmness \\
\hline CC & 27 & $50.12 \pm 2.61$ & $1.30 \pm 0.232$ \\
TC & 60 & $57.11 \pm 0.97$ & $1.79 \pm 0.085$ \\
TT & 84 & $56.19 \pm 0.78$ & $1.51 \pm 0.068$ \\
p value & - & $0.045^{*}$ & $0.015^{*}$ \\
CC-TT & - & $0.027^{*}$ & 0.37 \\
CC-TC & - & $0.014^{*}$ & $0.047^{*}$ \\
TC-TT & - & 0.455 & 0.010 \\
$*$ p $<0.05 * *$ p $<0.01$ & & &
\end{tabular}

The $1199 \mathrm{bp}$ cDNA sequence in pig ANKRD2 contains a 25 bp 5'UTR region which is different from a 209 bp size and a $51 \mathrm{bp}$ size, respectively in human and mouse, it encoded a 328 amino acid protein which was the same in length with mouse but distinguished from the human ANKRD2 composed of an extra 31 aa in the $\mathrm{N}$-terminal. It is suggested that divergent transcriptional and translational regulation mechanisms may work for the $A N K R D 2$ gene in mammals. ANKRD2 proteins with four ankyrin repeat in length of 30 amino acids were conservation in pig, mouse and human sequences. Ankyrin repeats within proteins are found in many different cellular sites both cytoplasmic and nuclear (Thompson et al., 1991). Researchers predicted porcine ANKRD2 protein might in both cytoplasmic and nuclear this structural feature is also presented in human and mouse which was connected with its functions. ANKRD2 in human, mouse and pig contains a single Protein-Destabilizing (PEST) sequence motif which might have been associated with their rapid degradation and enhance the activation of ANKRD2 and its transportation to the nuclear (McKinsey et al., 1997). Three Casein Kinase II (CK2) phosphorylation sites are constant architecture in the porcine ANKRD2 and happened to be conserved in pig, mouse and human. Interestingly, CK2 is a serine/threonine kinase that has been implicated in cell 
growth and proliferation (Bai et al., 2003). In human and mouse the promoter sequence of ANKRD2 aligned three boxes for muscle transcription factor, there were $\mathrm{MyoD}$, E47 and NF-kB (Bean et al., 2005). It suggests that the function of ANKRD2 is possibly related to CK and MyoD in skeletal muscle growth.

RT-PCR analysis demonstrated that porcine $A N K R D 2$ gene was expressed specially in skeletal muscle and heart. This result was consistent with observation form human and mouse that ANKRD2 specifically expressed in striated muscle. The expression levels increased gradually and reached the peak in adult, it revealed a fact that $\mathrm{ANKRD} 2$ may has a close relationship with muscle fiber development and will be up-regulated during the porcine muscle differentiation and hypertrophy period. The significant higher expressions in Landrance comparing with Toncheng pigs are observed in vary developmental stages through real-time PCR and suggested an important role for ANKRD2 in muscular hypertrophy (Kemp et al., 2000). There are higher intracellular muscular fat and small fiber size in Chinese indigenous pigs than in commercial pigs such as Landrace and large white. Landrace is well known for its high growth rate and lean meat percentage whereas Tongcheng pig has low growth rate and low lean meat percentage but more tenderness, juiced in characters. Thus, the expression profile of porcine ANKRD2 indicated that $A N K R D 2$ gene could associated with meat production which is affected by muscle fiber size and IMF.

ANKRD2 was previously mapped to porcine chromosome 14 and was linked to SW328 by radiation hybrid (ImpRH) panels (Dong et al. 2007). As reported, the Quantitative Trait Loci (QTL) on porcine chromosome 14 identified QTL for dressing percentage, loin muscle area and loin and neck meat weight which are located between the proximal part of SSC14 between SW540 and SW2488. SW328 is marked between SW540 and SW2488. Here, we provide evidence that a polymorphism in the $A N K R D 2$ gene was associated with the LEMM and Firmness traits which suggested ANKRD2 as a candidate gene of muscle development.

\section{CONCLUSION}

With respect to its physiological function in skeletal muscular development and controlling muscular hypertrophy and its position in the QTL region and association analysis as well as identified in the porcine, $A N K R D 2$ gene could possibly contribute to dissect these QTL or be the candidate gene/QTL itself and should thus be investigated in respective pedigrees. However, it is necessary to validate this association analyses in other population of pigs and it's essential that the dual functional character of ANKRD2 is proved through further study.

\section{ACKNOWLEDGEMENTS}

The researchers would like to thank the numbers of Dr Rothschild's Lab for their assistance on this research. The work was supported by the National High Technology Research and Development Program of China (863 Program, 2007AA1 0Z168) and the National Natural Science Foundation of China (30771536).

\section{REFERENCES}

Bai, Q., C. McGillivray, N. da Costa, S. Dornan, G. Evans, M.J. Stear and K.C. Chang, 2003. Development of a porcine skeletal muscle cDNA microarray: Analysis of differential transcript expression in phenotypically distinct muscles. BMC Genomics, 4: 8-8.

Bean, C., M. Salamon, A. Raffaello, S. Campanaro, A. Pallavicini and G. Lanfranchi, 2005. The Ankrd2, Cdknlc and calcyclin genes are under the control of MyoD during myogenic differentiation. J. Mol. Biol., 349: $349-366$

Bean, C., N. Facchinello, G. Faulkner and G. Lanfranchi, 2008. The effects of Ankrd2 alteration indicate its involvement in cell cycle regulation during muscle differentiation. Biochim. Biophys. Acta Mol. Cell Res., 1783: 1023-1035.

Cai, W., D.S. Casey and J.C. Dekkers, 2008. Selection response and genetic parameters for residual feed intake in Yorkshire swine. J. Anim. Sci., 86: 287-298.

Dong, X.J., H.P. Guan, Q.D. Zhang, M. Yerle and B. Liu, 2007. Mapping of porcine ANKRD1, ANKRD2, ANKRD23, VGLL2 and VGLL4 using somatic cell and radiation hybrid panels. Anim. Genet., 38: 424-425.

Hallenborg, P., S. Feddersen, L. Madsen and K. Kristiansen, 2009. The tumor suppressors pRB and p53 as regulators of adipocyte differentiation and function. Expert Opin. Ther. Targets, 13: 235-246.

Hicks, G.R. and N.V. Raikhel, 1995. Protein import into the nucleus: An integrated view. Ann. Rev. Cell Dev. Biol., 11: 155-188.

Inoue, N., N. Yahagi, T. Yamamoto, M. Ishikawa and $\mathrm{K}$. Watanabe et al., 2008. Cyclin-dependent kinase inhibitor, p21WAF1/CIP1, is involved in adipocyte differentiation and hypertrophy, linking to obesity and insulin resistance. J. Biol. Chem., 283: 21220-21229.

Kemp, T.J., T.J. Sadusky, F. Saltisi, N. Carey and J. Moss et al., 2000. Identification of Ankrd2, a novel skeletal muscle gene coding for a stretch-responsive ankyrin-repeat protein. Genomics, 66: 229-241. 
Kojic, S., E. Medeot, E. Guccione, H. Krmac and I. Zara et al., 2004. The Ankrd2 protein, a link between the sarcomere and the nucleus in skeletal muscle. J. Mol. Biol., 339: 313-325.

McKinsey, T.A., Z.L. Chu and D.W. Ballard, 1997. Phosphorylation of the PEST domain of IkappaBbeta regulates the function of NF-kappaB/IkappaBbeta complexes. J. Biol. Chem., 272: 22377-22380.

Molkentin, J.D. and E.N. Olson, 1996. Defining the regulatory networks for muscle development. Curr. Opin. Genet. Dev., 6: 445-453.

Moriyama, M., Y. Tsukamoto, M. Fujiwara, G. Kondo and C. Nakada et al., 2001. Identification of a novel human ankyrin-repeated protein homologous to CARP. Biochem. Biophys. Res. Commun., 285: 715-723.

Pallavicini, A., S. Kojic, C. Bean, M. Vainzof and M. Salamon et al., 2001. Characterization of human skeletal muscle Ankrd2. Biochem. Biophys. Res. Commun., 285: 378-386.
Panguluri, S.K., S. Bhatnagar, A. Kumar, J.J. McCarthy and A.K. Srivastava et al., 2010. Genomic profiling of messenger RNAs and microRNAs reveals potential mechanisms of TWEAK-induced skeletal muscle wasting in mice. PLoS One, 5: e8760-e8760.

Qiu, H., S. Zhao, X. Xu, M. Yerle and B. Liu, 2008. Assignment and expression patterns of porcine muscle-specific isoform of phosphoglycerate mutase gene. J. Genet. Genomics, 35: 257-260.

Thompson, C.C., T.A. Brown and S.L. McKnight, 1991. Convergence of Ets- and notch-related structural motifs in a heteromeric DNA binding complex. Science, 253: 762-768.

Tsukamoto, Y., N. Hijiya, S. Yano, S. Yokoyama and C. Nakada et al., 2008. Arpp/Ankrd2, a member of the muscle ankyrin repeat proteins (MARPs), translocates from the I-band to the nucleus after muscle injury. Histochem. Cell Biol., 129: 55-64. 